Y.-H. Chu, N.B. Suntzeff, J.E. Hesser, and D.A. Bohlender, eds.

\title{
Kinematic Study of the Northwestern Part of the Bar of the LMC
}

\author{
P. Ambrocio-Cruz, A. Laval, M. Marcelin, and P. Amram \\ Observatoire de Marseille. 2, Place Le Verrier, F-13248 Marseille \\ Cedex 04, France
}

\begin{abstract}
We have been conducting a Fabry-Perot kinematic survey of ionized gas in the Magellanic Clouds in the $\mathrm{H} \alpha$ and [O III] $\lambda 5007$ lines, using a 36-cm telescope at the European Southern Observatory. This poster presents our kinematic study of HII regions located at the northwestern parts of the LMC bar.
\end{abstract}

\section{The Survey}

An $\mathrm{H} \alpha$ and [OIII] $\lambda 5007$ survey of the Magellanic Clouds is being carried out at the European Southern Observatory in La Silla, Chile (Le Coarer et al. 1993 for the SMC; Laval et al. 1987 for the LMC). The instrument consists of a $36-\mathrm{cm}$ telescope with a focal reducer, a scanning Fabry-Perot interferometer, a photon counting camera, and a series of interference filters. We obtain images with a $38^{\prime} \times 38^{\prime}$ field of view, a $9^{\prime \prime}$ angular resolution, and a spectral sampling steps of 16 and $7 \mathrm{~km} \mathrm{~s}^{-1}$ for the $\mathrm{H} \alpha$ and [OIII] lines, respectively. The free spectral range is $377 \mathrm{~km} \mathrm{~s}^{-1}$ for $\mathrm{H} \alpha$ and $288 \mathrm{~km} \mathrm{~s}^{-1}$ for [OIII].

\section{The Nebulae}

In this poster we report on the sample of nebulae located on the northwest part of the LMC bar: DEM L117, DEM L115, DEM L107, DEM L99, DEM L98, DEM L97, DEM L89, DEM L76, N105, N103, N100, N33, N30, and N23. Table 1 shows the main characteristics of these nebulae. The linear diameters are derived by adopting a distance of $50 \mathrm{kpc}$ to the LMC (Feast 1991). Columns 3 and 4 give the systemic heliocentric velocities measured in $\mathrm{H} \alpha$ and [OIII]. The last column indicates whether the line profiles show splittings.

\section{Results}

Five of the surveyed objects show splittings in their line profiles. By calculating the energy injected into the gas we have concluded that the splittings in DEM L84 is caused by a supernova explosion (Ambrocio-Cruz et al. 1997). The peculiar velocity field of DEM L86 and its morphology indicate that it contains four bubbles and two bright compact HII regions, almost coeval, embedded inside a large, faint nebular shell. This structure is formed by a combination of 
Table 1. Main Characteristics of the Nebulae

\begin{tabular}{crrrr}
\hline Name of nebula & $\begin{array}{r}\text { Diameter } \\
(\mathrm{pc})\end{array}$ & $\begin{array}{r}V_{\mathrm{H} \alpha} \\
\left(\mathrm{km} \mathrm{s}^{-1}\right)\end{array}$ & $\begin{array}{r}V_{[O I I I} \\
\left(\mathrm{km} \mathrm{s}^{-1}\right)\end{array}$ & Splitting \\
\hline DEM L121 (N33) & 24 & 302 & - & no \\
DEM L117 & 22 & 306 & - & no \\
DEM L115 & 26 & 305 & - & no \\
DEM L107 & 126 & 304 & - & no \\
DEM L106 (N30) & 74 & 290,310 & - & yes \\
DEM L105 (N30) & 130 & 285,305 & - & yes \\
DEM L99 & 61 & 298 & - & no \\
DEM L98 & 68 & 305 & - & no \\
DEM L97 & 65 & 303 & - & no \\
DEM L89 & 131 & 293 & - & no \\
DEM L86 (N105) & 112 & 255 & 255 & yes \\
DEM L84 (N103) & 153 & 252 & 263 & yes \\
DEM L79 (N100) & 48 & 245 & - & no \\
DEM L76 & 153 & 264 & - & no \\
DEM L74 (N23) & 39 & 259,300 & - & yes \\
DEM L73 (N23) & 114 & 265 & - & no \\
DEM L70 (N23) & 81 & 265 & - & no \\
DEM L66 (N23) & 72 & 274 & 276 & no \\
\hline \hline
\end{tabular}

stellar winds and "champagne" outflows in a medium with a negative density gradient.

The line profiles observed in DEM L74 can be fitted by the sum of two Gaussians. The brighter component at $300 \mathrm{~km} \mathrm{~s}^{-1}$ is seen over the whole nebula and the fainter one at $259 \mathrm{~km} \mathrm{~s}^{-1}$ is seen only on the northwestern side. The intensity of this low-velocity component increases toward DEM L73 (located at northwest of DEM L74 and with a velocity of $265 \mathrm{~km} \mathrm{~s}^{-1}$ ). Thus, these splittings cannot be interpreted as caused by expansive motions. This lowvelocity component is most likely part of DEM L73 spilt over DEM L74.

The line profiles observed in DEM L105 and 106 can be fitted by the sum of two Gaussians. The first velocity component has a heliocentric velocity of 305$310 \mathrm{~km} \mathrm{~s}^{-1}$ and the second component has a velocity of $285-290 \mathrm{~km} \mathrm{~s}^{-1}$. Since the second velocity component is best detected at the bright parts along the edge of the N30 bubble, the line split cannot be interpreted as caused by expansive motions. This $285 \mathrm{~km} \mathrm{~s}^{-1}$ component is probably associated with a low-velocity HI component; it is possible that some UV flux of the blue supergiant stars of N30 escapes and contributes to the ionization.

Acknowledgments. This work is supported by a fellowship from DGAPAUNAM.

\section{References}

Ambrocio-Cruz, P., Laval, A., et al. 1997, A\&A, 319, 973

Ambrocio-Cruz, P., Laval, A., et al. 1998, A\&A, 339, 173

Feast, M. W. 1991, In: The Magellanic Clouds, IAU Symp. 148, p. 1

Laval A., Boulesteix, J., et al. 1987, A\&A, 175, 199

Le Coarer, E., Rosado, M., et al. 1993, A\&A, 280, 365 